\title{
Fordyce granules and hyperplastic mucosal sebaceous glands as distinctive stigmata in Muir-Torre syndrome patients: characterization with reflectance confocal microscopy
}

\author{
Giovanni Ponti ${ }^{1}$, Andrea Meschieri ${ }^{2}$, Annamaria Pollio ${ }^{3}$, Cristel Ruini ${ }^{2}$, Marco Manfredini ${ }^{2}$, Caterina \\ Longo $^{4}$, Victor D. Mandel $^{2}$, Silvana Ciardo ${ }^{2}$, Aldo Tomasi ${ }^{1}$, Luca Giannetti ${ }^{5}$, Giovanni Pellacani ${ }^{2}$ \\ ${ }^{1}$ Department of Diagnostic and Clinical Medicine and Public Health, University of Modena and Reggio Emilia, Modena, Italy; \\ ${ }^{2}$ Department of Dermatology, University of Modena and Reggio Emilia, Modena, Italy; ${ }^{3}$ Department of Neurosciences, University of \\ Padua, Padua, Italy; ${ }^{4}$ Dermatology and Skin Cancer Unit, Arcispedale S. Maria Nuova, IRCCS, Reggio Emilia, Italy; ${ }^{5}$ Department of \\ Paediatric Dentistry, University of Modena and Reggio Emilia, Modena, Italy
}

BACKGROUND: The Muir-Torre syndrome (MTS), a variant of Lynch syndrome (LS), is characterized by the presence of sebaceous skin adenomas and/or carcinomas and keratoacanthomas associated with visceral malignancies. Fordyce granules (FGs) are oral mucosal lesions previously found in association with LS. The aim of this study was to analyze the specific frequency of FGs in sporadic individuals and gene carriers patients with MTS of known mismatch repair genes mutations. The secondary aim was to characterize FGs by means of reflectance confocal microscopy (RCM).

METHODS: A total of 13 patients belonging to nine different genetically unrelated MTS kindreds (MLHI gene mutation $n=2 ; \mathrm{MSH} 2$ gene mutation $n=I I)$ and I40 genetically unrelated healthy controls were examined. Depending on the clinical examination of the oral mucosa surface, subjects were categorized as either FGs positive or FGs negative.

RESULTS: FGs were diagnosed in 13 of 13 (100\%) of MMR gene carriers patients with MTS vs. 9 of I40 (6.4\%) controls. The most common site for FGs in MTS was the vestibular oral mucosa, compared with the gingival mandibular and retromandibular pad in controls. RCM examination found multiple sebaceous acinar cells that appear as round or oval hyper-refractive globules and that create a lobular aspects of the sebaceous glands defined as 'moruliform' or 'berry-like' structures.

CONCLUSIONS: Clinical and RCM evidences of our study suggest that an activation of the sebaceous glands system occurs in patients with MTS. Fordyce granules

Correspondence: Dr. Giovanni Ponti, MD, PhD, Department of Clinical and Diagnostic Medicine and Public Health, University of Modena and Reggio Emilia, via del Pozzo, 41100 Modena, Italy. Tel: +39 059/4222600; Fax: +39 059/4222647; E-mail: giovanni.ponti@unimore.it

Accepted for publication July 24, 2014 and intra-oral sebaceous hyperplasia may constitute an additional clinical parameter, which may be adopted to distinguish individuals with highest likelihood of being affected from MTS.

J Oral Pathol Med (2015) 44: 552-557

Keywords: Fordyce granules; lynch syndrome; mismatch repair genes; Muir-Torre syndrome

\section{Introduction}

LS is characterized by the onset of visceral, mainly colorectal cancer (CRC), at an early age, and high frequency of synchronous and metachronous colonic and extracolonic tumors (gastrointestinal, endometrial, ovarian, pancreatic tumors, transitional cell carcinoma of the ureter and renal pelvis, and many more) $(1,2)$. MTS, a variant of LS, is characterized by the presence of sebaceous skin adenomas and/or carcinomas and keratoacanthomas associated with visceral malignancies (3). Sebaceous adenoma is composed of sebaceous lobules of various sizes that are incompletely differentiated, and contains basaloid cells at the periphery and mature sebaceous elements in the center. Sebaceous carcinoma is a malignant neoplasm with prominent cellular pleomorphism and anaplasia which most commonly occurs on the eyelids, where it generally arises from the meibomian glands or from the Zeiss glands $(4,5)$. Nearly half of patients with MTS had two or more visceral malignant diseases, $10 \%$ had more than four, and one individual had up to nine visceral cancers $(6,7)$.

FGs are oral mucosal lesions typically found in association with LS (8). The prevalence of FGs increases from childhood $(0.2 \%)$ (9) to adulthood $(0.97 \%)$ and is slightly higher in adult males $(1.77 \%)$ than females $(0.52 \%)$ 
(10). De Felice et al. (8) reported that an activation of the sebaceous gland system may occur in individuals carrying HNPCC gene mutations and/or patients fulfilling Amsterdam criteria. The authors stated that the frequency of recognizable FGs was approximately 50-90 times higher in HNPCC patients than in controls and suggested that this finding may help clinicians identify affected families (8).

FGs consist in intra-oral ectopic sebaceous glands and are considered benign lesions arising on the oral mucosa of adult subjects (Fig. 1). They can be found in different sites in the oral cavity, mainly at the upper lip vermilion, retromolar area, and buccal mucosa, and they are clinically characterized by minuscule yellowish macules or papules, the size of a pinhead. The clinical examination alone is enough to easily diagnose FGs, with no need for histopathological examination (11).

FGs should be differentiated from sebaceous glands hyperplasia ( $\mathrm{SGH}$ ) following the criteria proposed by Daley (1992) (8); histologically, more than 15 well-differentiated sebaceous lobules per gland suggest SGHs, while fewer than 15 lobules are interpreted as FGs.

Further research on larger populations is needed to confirm the reported clinical association and to prove the predictive value of FGs in detecting HNPCC and/or in MTS disorders; in particular, sebaceous skin neoplasms are the most distinctive features in MTS, and we can therefore hypothesize that similar to the skin, also the oral mucosa could exhibit an activation of the sebaceous gland system.

Nowadays, skin can be investigated and characterized through reflectance confocal microscopy (RCM), a noninvasive diagnostic tool that allows the evaluation of skin structures at a cellular resolution, constituting a biopsy 'in vivo.' While many studies exist on healthy skin and melanocytic and non-melanocytic lesions, very few studies have been performed on the characterization of mucosal lesions $(12,13)$. This is now possible thanks to the Vivascope $3000^{\circledR}$ (Vivascope $3000^{\circledR}$, Caliber I.D., Rochester, NY, USA). The instrument consists of a handheld probe that could be directly applied on the area of interest enabling high-resolution morphology evaluation of cells and structures in a fast and non-invasive procedure (14).

The aim of this study was to analyze the specific frequency of FGs in sporadic individuals and patients gene carriers of known MMR gene mutations with MTS phenotype. Moreover, the authors aimed to characterize intra-oral sebaceous glands by means of reflectance confocal microscopy (RCM) in patients with MTS and sporadic individuals.

\section{Methods}

Population

Inclusion criteria for patients with MTS were as follows: diagnosis of at least one sebaceous skin neoplasm synchronous or metachronous with at least one internal malignancy typical of LS; only patients with MTS who agreed to undergo MMR genetic testing, or for which the MMR germ line mutational status was already known were enrolled (11 MSH2 and 2 MLH1 germ line mutations; Table 2). Individuals with undetermined MMR mutational status were excluded.

The healthy control group included consecutive patients of both genders, Caucasian race, age $\geq 18$ years, who referred to dental outpatient clinic for the first time and exclusively for dental disease (tooth extraction, caries, periodontal disease), with no detectable oral mucosal lesions and no personal or family history of sebaceous skin tumors or internal malignancies.

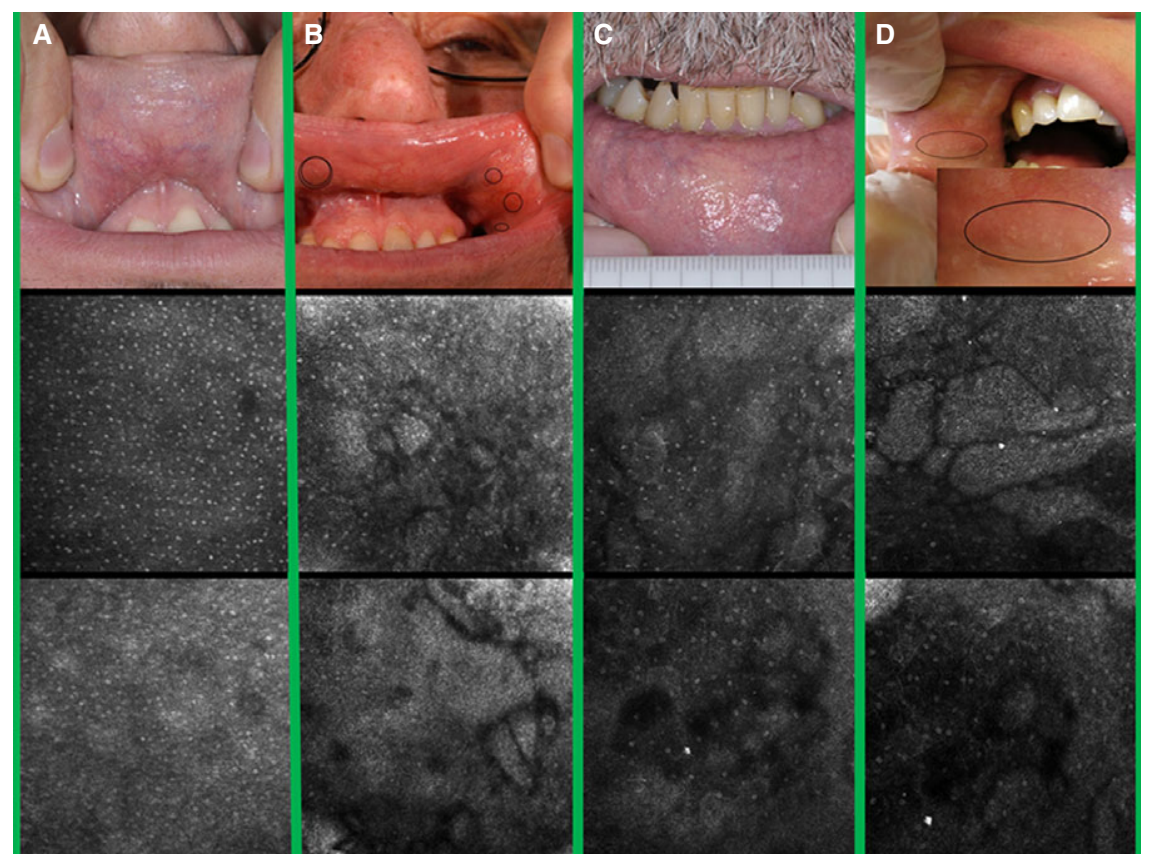

Figure 1 Clinical and reflectance confocal microscopy features of Fordyce granules in patients with Muir-Torre syndrome. (A) Clinical and RCM appearance of the oral vestibular mucosa in a healthy control subject, (B, C, D) Fordyce granules (FGs) and their RCM patterns in the oral vestibular mucosa of patient with MTS carrying the MMR germ line mutations. FGs appear as rice-like yellow-white papules surrounded by normal mucosa. 
One hundred forty healthy individuals (64 females and 76 males) with a mean age of $55.01 \pm 21.9$ years (CI: 51.3 58.7) and 13 gene carriers patients with MTS of MMR constitutional mutations (7 females and 6 males) of nine different genetically unrelated MTS kindreds (Tables 1 and 2) were included in the study; exhaustive clinical and anamnestic data were collected for each patient, while signed written informed consent was provided. Verification of cancer occurrence among family members was obtained in the majority of patients through clinical charts, pathologic records, or death certificates.

The oral mucosa surface was examined by two experienced odontostomatologists (Prof. Luca Giannetti and Dr. Annamaria Pollio) who classified as either FGs positive or FGs negative.

\section{Immunohistochemistry}

Immunohistochemical analysis of MLH1, MSH2, and MSH6 proteins was carried out on paraffin-embedded tumor samples. Immunoperoxidase staining using diaminobenzidine as a chromogen was run with the NEX-ES Automatic Staining System (Ventana, Strasbourg, France). The mouse monoclonal antibodies used were anti-MSH6 (clone 44; Transduction Laboratories) at 1:2000 dilution, anti-MLH1 (G168-15; Pharmingen) at 1:40 dilution, and anti-MSH2 (G129-1129; Pharmingen) at 1:40 dilution. Nuclei were counterstained with hematoxylin. Adjacent normal tissues from each sample served as positive controls.

\section{Mismatch repair genes mutational analysis}

Germ line mutations in $\mathrm{h} M L H 1, \mathrm{~h} M S H 2$, and $\mathrm{h} M S H 6$ were studied by single-strand conformation polymorphism (SSCP) on DNA derived from blood leukocytes. Samples that showed an altered SSCP mobility pattern were sequenced by means of the Sequenase ${ }^{\circledR}$ PCR product sequencing kit (Amersham Life Science, Buckinghamshire, UK) using a Beckman sequencer (model CEQ 8000). The exons of the MLH1 and MSH2/MSH6 genes from genomic

Table 1 Prevalence of Fordyce granules and neoplastic comorbidities in patients with Muir-Torre syndrome and healthy controls matched for age and sex

\begin{tabular}{|c|c|c|c|}
\hline & $\begin{array}{c}\text { Patients } \\
\text { with } \\
\text { Muir-Torre }\end{array}$ & $\begin{array}{l}\text { Healthy } \\
\text { control } \\
\text { group }\end{array}$ & $\begin{array}{c}P \text { value } \\
\text { (Fisher's exact } \\
\text { test }) \alpha<0.05\end{array}$ \\
\hline Patients (N\%) & $13(100 \%)$ & $140(100 \%)$ & \\
\hline Age & $\begin{array}{l}42.5 \pm 11.5 \\
(\text { CI } 35.6-49.5)\end{array}$ & $\begin{array}{l}55.01 \pm 21.9 \\
(\text { CI 51.3-58.7) }\end{array}$ & \\
\hline $\mathrm{F} / \mathrm{M}$ & $7 / 6$ & $64 / 76$ & \\
\hline $\begin{array}{l}\text { Patients with } \\
\text { Fordyce } \\
\text { granules (N\%) }\end{array}$ & $13(100 \%)$ & $9(6.4 \%)$ & $P<0.0001$ \\
\hline \multicolumn{4}{|c|}{ Tumors in patients } \\
\hline Breast & $1(7.7 \%)$ & $1(0.7 \%)$ & $P=0.16$ \\
\hline Colon & $7(53.8 \%)$ & $0(0 \%)$ & $P<0.0001$ \\
\hline Uterus & $2(15.4 \%)$ & $0(0 \%)$ & $P=0.007$ \\
\hline Kidney & $1(7.7 \%)$ & $0(0 \%)$ & $P=0.08$ \\
\hline Prostate & $1(7.7 \%)$ & $1(0.7 \%)$ & $P=0.16$ \\
\hline Brain & $0(0 \%)$ & $1(0.7 \%)$ & $P=1$ \\
\hline $\begin{array}{l}\text { Sebaceous } \\
\text { adenoma or } \\
\text { carcinoma } \\
\text { of skin }\end{array}$ & $8(61.5 \%)$ & $0(0 \%)$ & $P<0.0001$ \\
\hline
\end{tabular}

DNA were individually amplified and subsequently sequenced directly. Germ line mutations in MMR genes were defined according to standard nomenclature recommendations (http://www.hgvs.org/mutnomen/).

The study was approved by the local ethics committee in accordance with ethical standards on human experimentation and with the Declaration of Helsinki.

\section{Reflectance confocal microscopy (RCM)}

Dermoscopic images were recorded by means of a digital epiluminescence microscope (Vidix, MediciMedical, Castelfranco Emilia, Italy), using 20-fold, 30-fold, and a 50-fold magnification. Confocal imaging was collected using a handheld confocal laser scanning microscope (RCM) (Vivascope 3000, Caliber, Rochester, NY, USA), a noninvasive diagnostic tool that allows the real time examination of structures thanks to a diode laser at $830 \mathrm{~nm}$ and a power of $<16 \mathrm{~mW}$. RCM provides a horizontal optical resolution of $1.25 \mu \mathrm{m}$ and a vertical optical resolution of $5 \mu \mathrm{m}$ at the center of the field of view, acquiring single frames of $750 \times 750 \mu \mathrm{m}$ at a resolution of $1000 \times 1000$ pixels. A sequence of full-resolution individual images at a given depth was acquired for each lesion.

\section{Results}

FGs were diagnosed in 13 of $13(100 \%)$ MMR gene carriers patients with MTS vs. 9 of $140(6.4 \%)$ controls. The diameter of FGs ranged from 0.5 to $4 \mathrm{~mm}$ and the most common site for FGs being the vestibular oral mucosa in patients with MTS compared with the gingival and mandibular retromandibular pad in controls.

In the healthy control group of 140 patients (64 female and 76 male) with a mean age of $55.01 \pm 21.9$ years (CI: 51.3-58.7), only $9(6.4 \%)$ patients showed the presence of FGs localized in oral mucosa at clinical examination.

In patients with MTS, skin lesions were often multiple, synchronous, or metachronous, and were located predominantly in the head and neck. In this group, there were 23 skin lesions, including 22 sebaceous adenomas and 1 sebaceous carcinomas. The average age at onset of the first skin malignancy was 42.54 years (range 23-65 years). In eight patients with MTS, sebaceous lesions occurred as the first neoplasm while in the remaining five patients, these lesions developed after an internal malignancy. Among the 13 MTS probands, 7 colorectal carcinomas and 2 uterus carcinomas were found. The average age at onset of the first visceral malignancy was 45.71 years. In addition to skin and visceral malignancies, the tumor spectrum included one kidney carcinoma, one carcinoma of the renal pelvis, and one breast tumor.

\section{RCM features}

In reflectance confocal microscopy, the superficial layer of the epidermis of patients and healthy individuals (controls and with FGs) presents large cells with bright cytoplasm and small dark nuclei. Those cells appear hyporefractive because of the glycogen content and well demarcated because of the desmosomes. Although the superficial layer appears very similar in patients and controls, in the suprabasal layer of the epidermis, important differences can be found. 


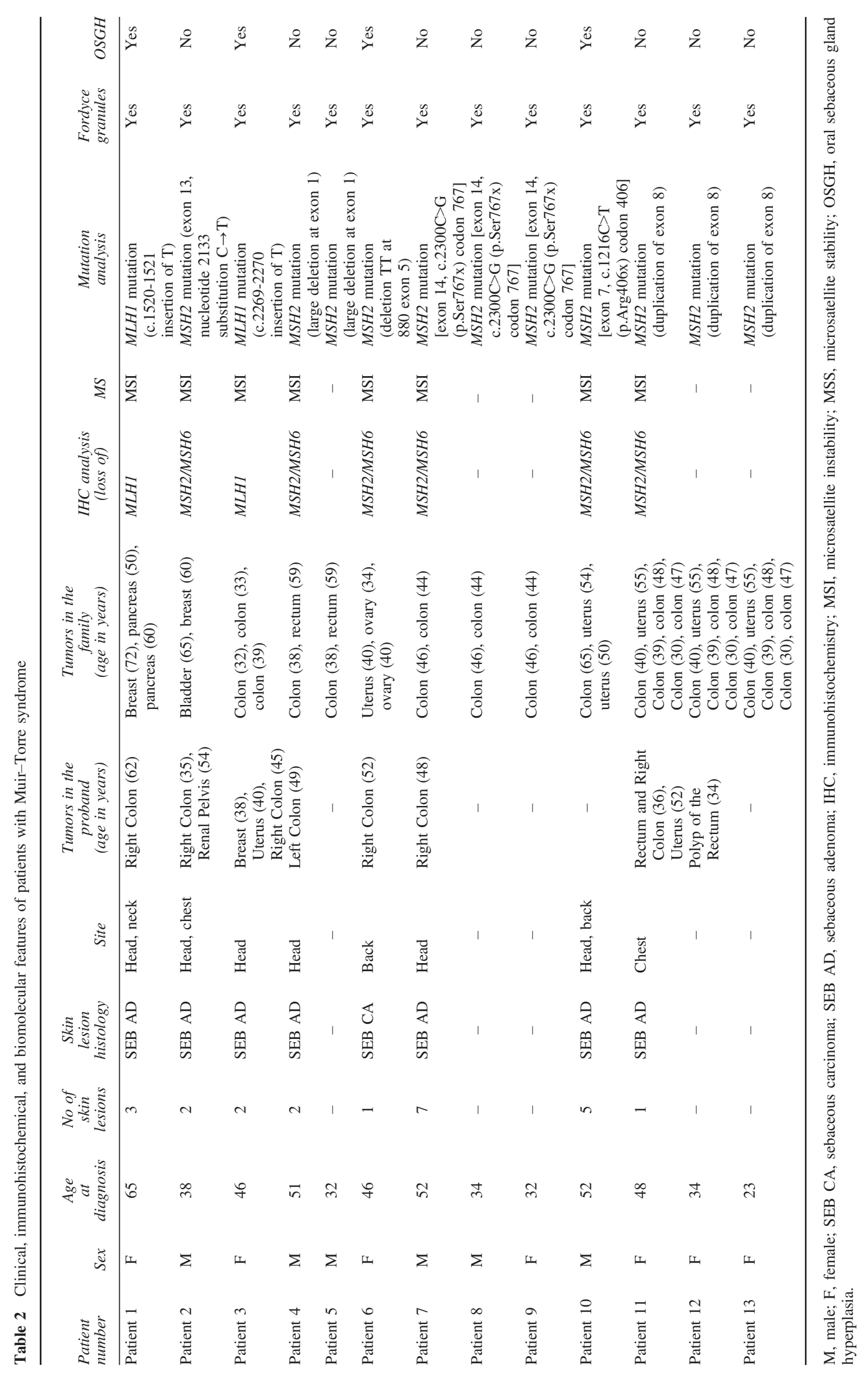


RCM images of subjects without FGs show the presence of small bright cells that create a sort of 'snowflake-like' pattern (Fig. 1a) while the suprabasal layer of the epidermis of a patient with FGs is characterized by the same rise-like pattern with the presence of bright lobular structures representing ectopic sebaceous glands, better seen in the deepest part of the oral mucosa (Fig. 1b,c).

At the dermoepidermal junction (DEJ), MTS patients with FGs have dark round dermal papillae with central bright linear or dotted vessels. In this context, we have found multiple sebaceous acinar cells that appear as round or oval hyper-refractive globules that create lobular aspects of the sebaceous glands defined as 'moruliform (berry-like) structures' (Fig. 1b,c).

On the other hand, the RCM analysis of individuals without FGs at DEJ shows only the presence of dark dermal papillae with central vessels and the absence of moruliform or berry-like structures typically seen in the ectopic sebaceous glands. In the superficial dermis of healthy subjects, it is possible to recognize bright hyper-reflective collagen fibers organized in bundles and linear or hairpin vessels that appear as hyper-refractive tubular structures (Fig. 1a). In MTS patients with FGs, RCM examination displays bright collagen fibers, canalicular vessels, and also moruliform or berry-like structures corresponding to multiple sebaceous glands located in depth.

In case of sebaceous hyperplasia, detected in association with FGs in one-third of MTS probands, the numbers of the gland's lobules at the DEJ increase (Table 2); the lobules are well demarcated and hyper-refractive and become more visible not only at the DEJ, but also in most superficial epidermal layer (Fig. 1d).

\section{Discussion}

The main clinical evidences of our study establish an important connection between FGs and gene carriers patients with MTS of known MMR gene mutations. Our study reveals a FGs frequency rate of $100 \%$, which is even higher than the rate of $86.7 \%$ reported in LS by the previous study that inspired our epidemiological investigation (8). In addition to research and evaluation of FGs in the cohort of patients with MTS, we evaluated their frequency in a control population, reporting an incidence of $6.4 \%$. FGs are sufficiently uncommon in the general population to consider them as potential markers of MTS. The MTS is, in fact, a clinical variant of the LS; the two entities share the same biomolecular pathogenesis, due to constitutional mutations of MMR genes (15). The main difference concerns the neoplastic phenotype, which includes in MTS cutaneous sebaceous neoplasms. The MTS may therefore be considered as the full phenotypic expression of LS; however, the reason for an increased susceptibility to the development of sebaceous neoplasms in certain families is not yet known, due to the lack of a specific genotype-phenotype correlation. Our hypothesis supporting the clinical role of FGs within MTS, as it is in LS, could be confirmed by the knowledge that, in this setting, skin appendages, and in particular sebaceous glands, typically tend to both hyperplastic and/or clearly neoplastic transformation (sebaceous adenomas and carcinomas) (16).
Our evidences suggest that FGs are not extremely common in the general population; moreover, we believe that from a qualitative point of view, clinical pictures of sebaceous glands hyperplasia and/or multiplicity of ectopic glands can be significantly associated with the MTS phenotype.

From a clinical perspective, it is important to collect more elements to identify whether patients who present with sebaceous tumors are in need of further biomolecular and immunohistochemical evaluation. At this regard, a clinical scoring system has been recently proposed to recognize which patients with sebaceous neoplasms can be at risk for MTS, including clinical-anamnestic variables: in particular, onset age of sebaceous neoplasms, total number of sebaceous neoplasms, personal and/or family history of LS-related malignancies (17). We believe that the search for FGs may constitute an additional clinical parameter, which should certainly be confirmed in a wide MTS patient cohort, but which will be adopted as a novel variable to recognize individuals with the highest likelihood of having MTS.

Daley's criteria defined the difference between FGs and mucosal sebaceous hyperplasia, adopting a minimum cutoff of 15 lobules (11); however, an important limit consists in the need for histopathology, so that a biopsy of the lesion is necessary but not always clinically justified. These criteria could be improved by including RCM characterization; in vivo RCM examination may allow diagnostic differentiation between ectopic and hyperplastic glands just based on the quantitative evaluation of the different components of gland lobules. It is clear that the RCM approach would avoid the issue of a histology-based diagnostic definition for lesions that do not usually require biopsy and/or surgical excision.

To date, there are no other studies regarding the diagnostic characterization of mucosal sebaceous glands by RCM: our innovative approach has shown that it is possible to define a characteristic image pattern of ectopic sebaceous glands of the oral mucosa that partially overlaps with those of the skin (18). In particular, the snowflakes texture of the mucosal surface, expression of nucleated keratinocytes, characterizes the entire field in which we distinguish some groups of acinar structures with moruliform or berry-like appearance; those are clearly visible also in the deeper dermal layers, probably due to the absence of the superficial keratinized layer. In fact, it is known that the mucous membranes in the superficial layer show large illdemarcated cells with prominent nucleus, while the suprabasal layers show large cells with small nucleus, which are hyporefractive (because of the glycogen content) and well demarcated (because of desmosomes).

It is important to underline that a few sebaceous structures can be visible in both healthy and FGs mucosa; those appear as small hyper-refractive cell clusters and become therefore better visible while the gland becomes ectopic and, at the same time, more superficial. This can be due to a sort of progression from single sebaceous cells, to glandular ectopy, to hyperplasia (Fig. 1).

There are therefore evidences in favor of a link between ectopic sebaceous gland and MMR genes' defects. The biological significance of this association remains, to date, unclear. Hedgehog signaling pathway has been shown to increase the size and number of sebaceous glands $(19,20)$; 
the authors of this study suggest that the activation of $\mathrm{Hh}$ pathway may coexist in HNPCC/MTS, leading to development of FGs. In the end, we hypothesize that the activation of the sebaceous glands' system occurs in patients with MTS. This hypothesis is supported by the immunohistochemical and molecular characterization that allowed us to confirm the molecular pathogenesis in all patients with MTS enrolled in the study. All patients carried specific MMR mutations. In particular, our cohort highlights the predominant role of $\mathrm{MSH} 2$ (11 cases) mutations compared to MLH1 (2 cases) in the pathogenesis of MTS phenotype (Table 2).

FGs and/or sebaceous hyperplasia present in the oral mucosa may be a clinical marker associated with MTS and represent a clinical stigmata of the syndromic heredofamilial spectrum. The relatively low frequency of FGs in the general population supports the potential use of such lesions in the identification of MTS, as well as in the recognition of potential gene carriers of germ line mutation within specific families that can be selectively addressed to genetic screening.

RCM is a non-invasive diagnostic tool that allows us to characterize FGs from the diagnostic point of view without the need of a biopsy; in particular, it provides a quantitative and qualitative assessment of glandular acini of the mucosa and the collection of specific image patterns of gland hyperplasia.

Future studies on larger populations carrying MMR gene mutations are needed to clarify the predictive value of FGs in the detection of MTS and HNPCC.

\section{References}

1. Lynch HT, Smyrk T. Hereditary nonpolyposis colorectal cancer (Lynch syndrome). Cancer 1996; 78: 1149-67.

2. Bellacosa A, Genuardi M, Anti M, et al. Hereditary nonpolyposis colorectal cancer: review of clinical, molecular genetics, and counseling aspects. Am J Med Genet 1996; 62: 353-64.

3. Cohen PR, Kohn SR, Davis DA, et al. Muir-Torre syndrome. Dermatol Clin 1995; 13: 79-89.

4. Gloor P, Ansari I, Sinard J. Sebaceous carcinoma presenting as a unilateral papillary conjunctivitis. Am J Ophthalmol 1999; 127: 458-9.

5. Dixon RS, Mikhail GR, Slater HC. Sebaceous carcinoma of the eyelid. J Am Acad Dermatol 1980; 3: 241-3.

6. Coldron J, Reid I. Muir-Torre syndrome. J R Coll Surg Edinb 2001; 46: 178-9.

7. Ponti G, de Leon MP. Muir-Torre syndrome. Lancet Oncol 2005; 6: 980-7.

8. de Felice C, Parrini S, Chitano G, et al. Fordyce granules and hereditary non-polyposis colorectal cancer syndrome. Gut 2005; 54: 1279-82.
9. Sedano HO, Carreon Freyre I, Garza de la Garza ML, et al. Clinical orodental abnormalities in Mexican children. Oral Surg Oral Med Oral Pathol 1989; 68: 300-11.

10. http://www.maxillofacialcenter.com/BondBook/softtissue/ford aycegran.html (Accessed on 23 June, 2005).

11. Daley TD. Intraoral sebaceous hyperplasia. Diagnostic criteria. Oral Surg Oral Med Oral Pathol 1993; 75: 343-7.

12. Ocampo-Candiani J, Villarreal-Rodríguez A, QuiñonesFernández AG, et al. Treatment of Fordyce spots with $\mathrm{CO} 2$ laser. Dermatol Surg 2003; 29: 869-71.

13. Debarbieux S, Perrot JL, Erfan N, et al. Reflectance confocal microscopy of mucosal pigmented macules: a review of 56 cases including 10 macular melanoma. Br J Dermatol 2013; 170: $1276-84$.

14. Manfredini M, Mazzaglia G, Ciardo S, et al. Does skin hydration influence keratinocyte biology? In vivo evaluation of microscopic skin changes induced by moisturizers by means of Reflectance Confocal Microscopy. Skin Res Tech 2013; 19: 299-307.

15. Ponti G, Losi L, Pedroni M, et al. Value of MLH1 and MSH2 mutations in the appearance of Muir-Torre syndrome phenotype in HNPCC patients presenting sebaceous gland tumors or keratoacanthomas. J Invest Dermatol 2006; 126: 2302-7.

16. Ponti G, Losi L, di Gregorio C, et al. Identification of MuirTorre syndrome among patients with sebaceous tumors and keratoacanthomas: role of clinical features, microsatellite instability, and immunohistochemistry. Cancer 2005; 103: $1018-25$

17. Roberts ME, Riegert-Johnson DL, Thomas BC, et al. A clinical scoring system to identify patients with sebaceous neoplasms at risk for the Muir-Torre variant of Lynch syndrome. Genet Med 2014. doi: 10.1038/gim.2014.19 [Epub ahead of print].

18. Hofmann-Wellenhof R, Pellacani G, Malvehy J, Soyer HP. Reflectance confocal microscopy for skin diseases, Berlin: Springer-Verlag, 2012.

19. Allen M, Grachtchouk M, Sheng H, et al. Hedgehog signaling regulates sebaceous gland development. Am J Pathol 2003; 163: $2173-8$.

20. Lum L, Beachy PA. The Hedgehog response network: sensors, switches, and routers. Science 2004; 304: 1755-9.

\section{Acknowledgements}

The authors have no conflict of interests relevant to this article to disclose. Honorarium, grant, or other form of payment was not given to anyone of the authors to produce the manuscript. All authors made substantive intellectual contributions to the published study, and each author listed on the manuscript has seen and approved the submission of the manuscript. We would like to express our gratitude to Prof. Percesepe Antonio (Medical Genetics, Department of Medical and Surgical Sciences, University Hospital of Modena, Italy), Dr. Mazzaglia Giovanna (MD, Department of Dermatology, University of Modena and Reggio Emilia, Modena, Italy), and Dr. Farnetani Francesca (MD, Department of Dermatology, University of Modena and Reggio Emilia, Modena, Italy) who gave valuable contributions for the article. 